\title{
Pulse Volume Recording Disturbances in Paraplegic Patients
}

\author{
R. Walden, MD, ${ }^{1}$ A. Bass, $M D,{ }^{1}$ A. Ohry, $M D,{ }^{2}$ J. Schneiderman, $M D,{ }^{1}$ \\ R. Adar, MD ${ }^{1}$ \\ ${ }^{I}$ The Lubinski Vascular Institute, Department of General and Vascular Surgery, \\ ${ }^{2}$ Department of Neurological Rehabilitation, Sheba Medical Center, Tel Hashomer, \\ and the Tel Aviv University Medical School, Isrseal.
}

\section{Summary}

Spinal cord injuries may modify vascular reactivity in the denervated region. This study presents an original observation of altered vascular response in paraplegic patients. A group of 30 consecutive paraplegic otherwise normal individuals underwent a thorough vascular examination. There were 29 male and 1 female patient, 21 to 67 years old (average 41), all with traumatic spinal cord injury. Average time since injury was 17 years. All had good peripheral pulses and normal segmental Doppler pressure measurements. In 8 patients, plethysmography_pulse volume recording (PVR) was normal as expected. In 22 patients an unusual feature of the vascular examination was recorded, consisting of normal peripheral arterial pressures with PVR waveforms indicating poor pulsatility. This group was older than the group with normal studies: ages $44 \pm 13$ vs $34 \pm 8$ years $(p=0.05)$, and more time had elapsed since injury-20 \pm 12 vs $10 \pm 4$ years $(p=0 \cdot 015)$. The altered pulsatility demonstrated in most of those paraplegic patients may play a role in deficient wound healing frequently observed below the level of spinal neurological loss.

Key words: Paraplegia; Pulse volume recording (plethysmography); Blood circulation; Blood pressure determination.

Spinal cord injuries resulting in upper or lower motor neuron lesions may modify vascular reaction in the denervated region. This study presents an original observation of altered vascular response in the lower extremities of paraplegic patients. These changes may play a role in the deficient would healing capabilities frequently observed below the level of spinal neurological loss. 


\section{Patients and methods}

Thirty paraplegic patients were examined in the vascular laboratory as part of a routine prospective study. There were 29 males and 1 female (patient no. 9) patients 21 to 67 (average 41 years) years old. All patients had traumatic spinal cord injury. The time since injury was 1 to 40 (average 17) years. The level of spinal cord injury varied: 7 had cervical, 20 had dorsal and 3 had lumbar. All had complete motor paralysis. Fifteen patients had upper motor neuron lesions and 15 had lower motor neuron lesions. All but one patient had no vascular symptoms or signs. The one patient with symptoms was the lady who presented with ulcerations of the first toe bilateraly. None of them was diabetic.

The non-invasive work up included: (1) a thorough medical history with emphasis on vascular symptoms; (2) physical examination with grading the strength of the lower extremity pulses; (3) measurements of the Doppler systolic opening pressure at the thigh, calf and ankle. From these measurements a pressure index was calculated by dividing the segmental pressure by the highest brachial pressure;

I

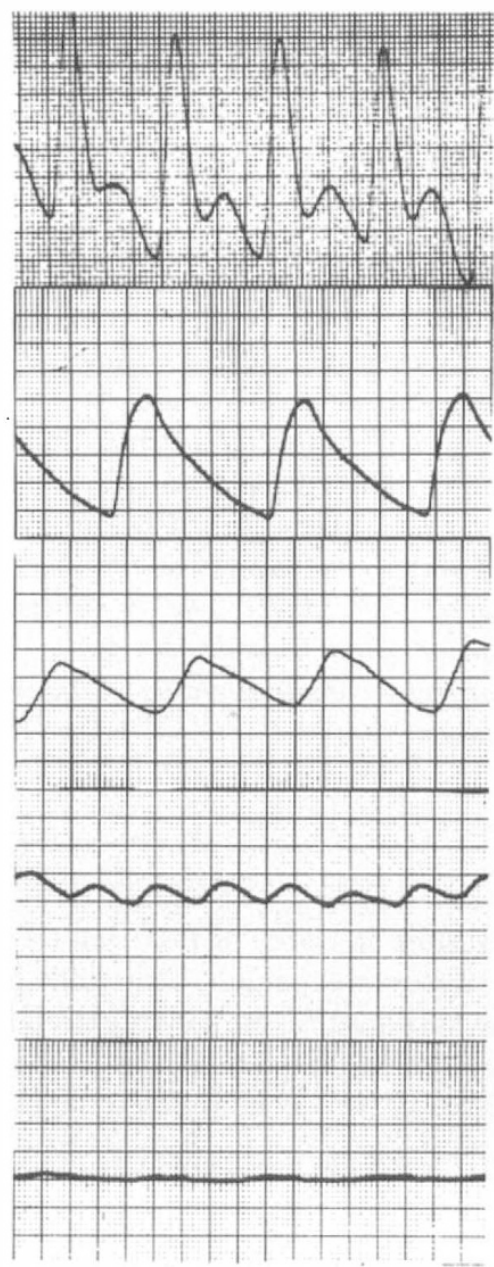

Figure 1 Pulse volume recording categories. 
and (4) plethysmography_-pulse volume recording (PVR-Life Sciences Co.) at the thigh, calf and ankle levels. PVR results were divided into five categories (Raines, 1976), as presented in Figure 1: I-Amplitude $>15 \mathrm{~mm}$ with a dicrotic notch; II-Same as I but without dicrotic notch; III-Amplitude between 5 and 15 mm; IV-Amplitude $<5 \mathrm{~mm}$ but clearly pulsatile; V-Flat line. Doppler pressure measurements and PVR are parallel indications of blood flow to an extremity. The exception is falsely high pressures recorded when severely calcified arteries do not collapse under high cuff pressures, as frequently seen in advanced diabetic peripheral vascular disease. Statistics-Mann Whitney rank sum test was used for statistical analysis.

\section{Results}

All patients had normal peripheral pulses and Doppler arterial pressures and indices. In 8 patients the PVRs were normal as well (Table I, Figure 2). The aver-

Table I Patients with normal pulse volume recording

\begin{tabular}{lcccc}
\hline No & Age & $\begin{array}{c}\text { Years since } \\
\text { injury }\end{array}$ & $\begin{array}{c}\text { Level of } \\
\text { lesion }\end{array}$ & $\begin{array}{c}\text { Motor neuron } \\
\text { lesion }\end{array}$ \\
\hline 1 & 30 & 10 & D10-12 & lower \\
2 & 29 & 10 & D10-12 & lower \\
3 & 31 & 11 & C6-7 & upper \\
4 & 41 & 16 & D4 & upper \\
5 & 30 & 12 & C5 & upper \\
6 & 50 & 7 & D11 & lower \\
7 & 36 & 10 & D11 & lower \\
8 & 23 & 3 & C3 & upper \\
\hline Mean & & & & \\
\pm SD & $34 \pm 8$ & $10 \pm 4$ & &
\end{tabular}
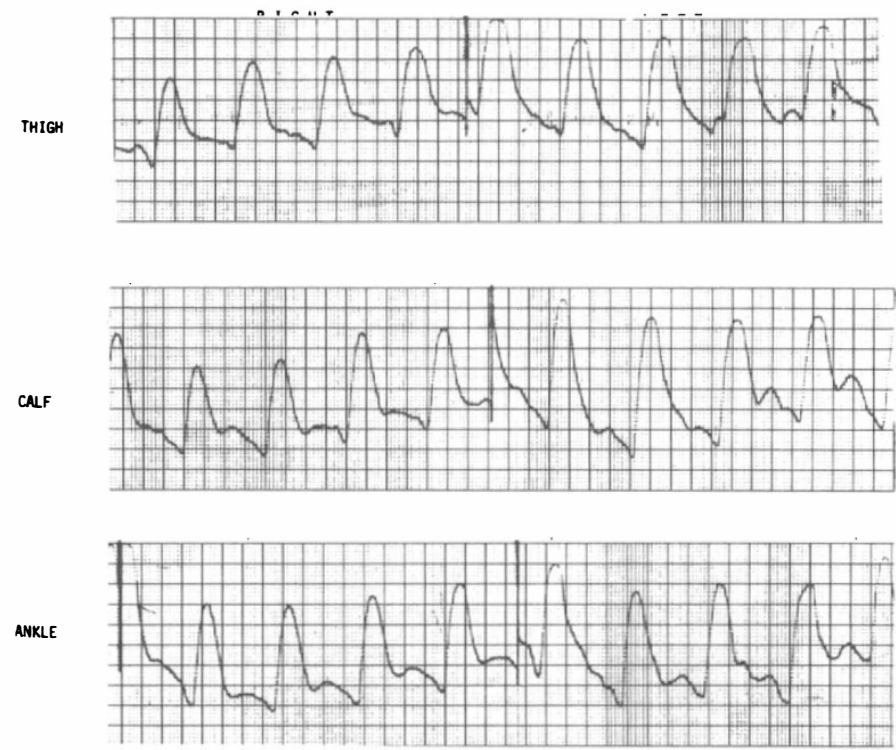

Figure 2 Normal pulse volume recording in a paraplegic patient (patient no. 1). 
Table II Patients with abnormal pulse volume recording

\begin{tabular}{rrccc}
\hline No & Age & $\begin{array}{c}\text { Years since } \\
\text { injury }\end{array}$ & $\begin{array}{c}\text { Level of } \\
\text { lesion }\end{array}$ & $\begin{array}{c}\text { Motor neuron } \\
\text { lesion }\end{array}$ \\
\hline 9 & 38 & 13 & L4 & lower \\
10 & 21 & 1 & L2-3 & lower \\
11 & 57 & 26 & D11-12 & lower \\
12 & 56 & 24 & D5 & upper \\
13 & 33 & 14 & D12-L1 & lower \\
14 & 36 & 16 & D3 & upper \\
15 & 29 & 8 & D12 & lower \\
16 & 52 & 32 & D10 & upper \\
17 & 43 & 22 & C6 & upper \\
18 & 35 & 11 & D7 & upper \\
19 & 42 & 20 & C6 & upper \\
20 & 60 & 40 & C6 & upper \\
21 & 35 & 4 & D8 & upper \\
22 & 60 & 40 & L3-5 & lower \\
23 & 26 & 5 & C7 & upper \\
24 & 52 & 31 & D10 & lower \\
25 & 67 & 40 & D12-L1 & lower \\
26 & 36 & 16 & D12 & lower \\
27 & 57 & 14 & D12 & lower \\
28 & 51 & 31 & D9 & upper \\
29 & 35 & 15 & D6 & upper \\
30 & 35 & 17 & D11-L1 & lower \\
\hline Mean & & & & \\
\pm SD & $44 \pm 13$ & $20 \pm 12$ & & \\
\hline
\end{tabular}
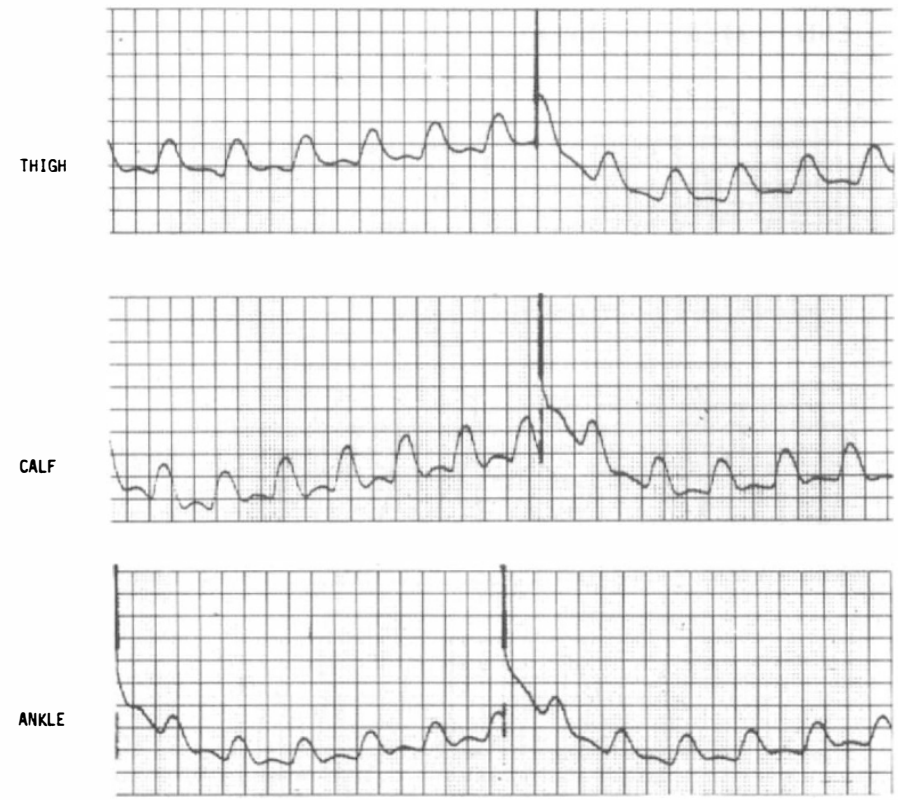

Figure 3 Typical pulse volume disturbance with poor pulsatility as observed in most paraplegics (patient no. 13). 
age age of these patients was 34 years and the average time since injury was 10 years. Three of them had cervical lesions and 3 had dorsal lesions. Four patients had upper motor neuron lesions and 4 had lower motor neuron lesions.

In 22 patients, an unusual feature of the vascular examination was observed with PVR waveforms indicating poor pulsatility (PVR categories III-IV) in the presence of good distal pulses and arterial pressures (Table II, Figure 3). In this group, the average age was 43 years and the average time since injury was 20 years. Four patients had cervical lesions, 15 had dorsal lesions and 3 had lumbar lesions. Eleven patients had upper motor neuron lesions and 11 had lower motor neuron lesions.

Patients in the group with disturbed flow patterns therefore differed from those with normal studies in older age $(p=0.05)$ and longer duration of paralysis $(p=$ $0.015)$. Differences in levels of lesions did not reach statistical significance. The distributions between the types of motor neuron lesions was equal between the groups and within the groups.

\section{Discussion}

Paraplegic patients with decubitus ulcers are particularly prone to delayed and complicated wound healing. This has been usually related to their immobility and sensory loss (Localio, 1958). In a recent study by Basson and Burney (1982) on paraplegic and quadriplegic patients, a high frequency of poor wound healing was found in nondecubitus wounds. They found a 5-fold increase in the incidence of wound healing complications in wounds occurring below the level of the spinal insult as compared with those above that level and with matched controls. Several mechanisms have been suggested as responsible for deficient wound healing in paraplegics. These include decreased macromolecular synthesis (Malman, 1979), altered collagen metabolism with increased excretion of collagen metabolites (Claus-Walker, 1979; Malman, 1979) and abnormal wound tension due to motor denervation (Basson, 1982). Brief mention is made of autonomic denervation with altered vascular responses as a possible relevant factor (Basson, 1982; Olivari, 1972), but with little evidence to support this notion in connection with wound healing.

In our study, 22 out of 30 patients presented a uniform decrease of PVR amplitude throughout the lower extremities in the presence of normal distal arterial pressures. This discrepancy is not unusual in diabetic patients with severely calcified noncompressible arteries. It was a completely unexpected finding in our series of relatively young, non-diabetic patients. The older age and longer time since injury in the group with decreased pulsatility compared with the one with normal studies, may indicate an effect of time in development of the abnormality. Muscle atrophy due to limb disuse may be a factor but this process should have been already quite advanced in the normal group with an average of 10 years since injury. We found no significant correlation between the decreased pulsatility and the level of spinal injury or the presence of upper or lower motor neuron lesion. Pulse volume recording closely parallels the actual pattern of blood flow into an extremity (Cambria, 1983). Our findings seem to indicate an alteration in the normal pulsatility pattern in the majority of paraplegic patients in this series. Organ systems depend on pulsatile flow for optimal perfusion (Baird, 1976). Experimen- 
tal modifications of arterial flow from pulsatile to steady-state lead to a rise in arterial pressure and peripheral resistance with reduction in brain oxygen consumption and excretory capacity of the kidney (Giron, 1966). Quite why flow has to be pulsatile is not clear; the regulating mechanism is not sympathetically mediated since it is not abolished by alpha adrenergic blockade with phenoxybenzamine (Giron, 1966).

These preliminary observations will need confirmation by further clinical and experimental studies. Yet, it seems that altered pulsatility may play a role in the mechanism responsible for deficient wound healing below the level of complete spinal neurological loss.

\section{References}

BAIRD RN, AвBOtт WM 1976 Pulsatile flow in arterial grafts. Lancet ii:948-950.

BASSON MD, BURNEY RE 1982 Defective wound healing in patients with paraplegia and quadriplegia. Surgery, Gynecology and Obstetrics 155:9-12.

Cambria RP, Walden R, Megerman J, L'Italien G, AbBott WM 1983 Noninvasive intraoperative assessment of arterial reconstruction. Archives of Surgery 1152-1156.

Claus-Walker J, Carter RE, Campos RJ, SPEnCER WA 1979 Sitting, muscular exercise and collagen metabolism in paraplegia. American fournal of Physical Medicine 58:287-293.

Giron F, BirRWELl WC, SorofF HS, DETERLING RA 1966 Hemodynamic effect of pulsatile and nonpulsatile flow. Archives of Surgery 93:802-810.

LoCALIO SA, LOMAN EW, GIBSON J 1958 Wound healing in the paraplegic patient. Surgery 44: 625-626.

MALMAN ML, DRESDEN MH 1979 Denervation effects on newt regeneration, collagen and collagenase. Developmental Biology 71:60-70.

Olivari N, Schrude J, WaHLE H 1972 The surgical treatment of bedsores in paraplegics. Plastic and Reconstructive Surgery 50:477-482.

Raines JK, Darling DC, Buth J, Brewster DC, Austin WG 1976 Vascular laboratory criteria for the management of the lower extremities. Surgery 79:21-29. 